\title{
Pseudocontingencies can override genuine contingencies between multiple cues
}

\author{
KLAUS FiEDLER \\ University of Heidelberg, Heidelberg, Germany
}

\begin{abstract}
The cognitive process of contingency assessment has traditionally been conceived as an inference from the joint frequencies (table cell entries) of correlated cues. Alternatively, pseudocontingency (PC) inferences are derived from the alignment of separate base rate trends (table marginals). The present research extends PCs to the simultaneous assessment of six contingencies between four personality cues. Consistently obtained PC effects, reflecting accurately assessed base rate trends, were unaffected by the actual cue intercorrelations, which were zero or consistent or inconsistent with the PCs. The functional value of PC inferences is discussed. Although PCs can be misleading, they afford approximations of actually existing correlations that suffice under many, although not under all, conditions.
\end{abstract}

Cognitive illusions must not be disregarded as blunt deficits of the fallible human mind. They often function as useful inference schemes in their original learning context and lead to erroneous inferences only when carried over to new environments. This insight pertains to the pseudocontingency (PC) illusion in particular (Fiedler \& Freytag, 2004; Fiedler, Freytag, \& Meiser, 2009). The affix pseudo refers to the fact that a contingency between two dichotomous variables, or a correlation between any pair of variables, is inferred from information that does not fit the logic of a correlation. In the absence of information about the joint occurrence of two variables $\left(x_{i}\right.$ and $\left.y_{i}\right)$ in the same individuals $i$ in the cells of a contingency matrix, correlations are readily inferred from unequal base rates in the marginals of the matrix. When both distributions are skewed in the same direction (both variables taking mostly high or mostly low values), the inferred correlation is positive. When skewed in opposite directions (toward high values on one and low values on the other variable), the inferred correlation is negative.

At first sight, PC inferences appear to be seriously flawed; it is a category mistake to infer a correlation from unequal base rates. If, for instance, in a foreign country, most people are vegetarian and most people are religious, the rate of vegetarians among religious people may be higher than, equal to, or lower than that among nonreligious people, yielding a positive, zero, or negative correlation, respectively. Just as two main effects, for rows and columns of a $2 \times 2$ design, do not constitute an interaction, the base rates of a contingency table do not define a correlation.

Nevertheless, when no joint observations about eating and religion are available, PCs afford a useful heuristic for inferring the correct sign of the correlation. If both vegetarians and religious people are prevalent in a particu- lar country, this may reflect a common cause. Bayesian statistics shows that the posterior probability of a positive (negative) correlation increases when $X$ and $Y$ are known to be skewed in the same (opposite) direction. Indeed, recent Monte Carlo simulations by Freytag, Kutzner, Vogel, and Fiedler (2010) demonstrated that PC affords a proxy for estimating actual correlations. PCs were computed for all possible $2 \times 2$ contingencies that resulted from the combination of four cell frequencies varying between 1 and 20 . When the marginal frequencies were skewed enough - that is, when the odds of the row frequencies and the column frequencies were at least $2: 1,3: 1$, or $4: 1$ - the PC indicated the correct sign of the correlation in $54 \%, 73 \%$, and $89 \%$ of all cases, respectively. Considering only substantial correlations exceeding $\Phi=.2$, these sign detection rates increased to levels as high as $62 \%, 95 \%$, and $100 \%$.

Like every illusion, PCs can produce seriously wrong inferences under some conditions (e.g., when nonvegetarians are particularly likely to be religious), but they provide useful information in many other situations. PC inferences are particularly valuable and plainly indispensable when, in reality, genuine correlation data are missing, as is often the case. Unlike statistics textbooks that presuppose $k$ joint measures for each of $n$ individuals, the information environment rarely provides us with such convenient data arrays. In the example, we might observe the high rates of vegetarians and religious people on different occasions, unable to link them to the same individuals.

In multivariate problem contexts with $k>2$ variables, the problem of assessing all intercorrelations can become immense. The number of missing values is typically large. Even when a full multivariate array exceptionally happens to be available, memory restrictions prevent us from assessing all $k \cdot(k-1) / 2$ contingencies between $k$ variables

K. Fiedler, kf@psychologie.uni-heidelberg.de 
at the same time. Such multiple-cue tasks should make PC inferences particularly likely. Whereas a genuine correlation assessment is hardly ever possible in such demanding task environments, PC-type inferences can be accomplished quite easily. Detecting clear-cut base rate trends in $k$ variables is sufficient to make PC inferences between all variables. In the absence of better information, relying on PCs would thus appear to be a reasonable strategy.

The present study is the first to investigate PC inferences in a multicue paradigm that calls for the learning of an entire correlation pattern. Previous research has been confined to the elementary case of PC inferences between only two focal variables, although PC effects have been obtained in both simple and complex task settings, using both dichotomous and continuous stimulus variables (see Fiedler et al., 2009). PCs have been observed for the assessment of correlations in personality tests (Fiedler \& Freytag, 2004), groups and stereotypically associated behaviors (Freytag, 2003; Meiser \& Hewstone, 2004), evaluation of student achievement (Fiedler, Freytag, \& Unkelbach, 2007), and operant feedback learning (Kutzner, Freytag, Vogel, \& Fiedler, 2008). Studies relied not only on explicit judgments of contingencies, probabilities, or frequencies, but also on implicit measures of PCs, such as online predictions or cued recall performance. In recent speededclassification experiments, response latencies in evaluative priming (Fiedler, Freytag, \& Bluemke, 2010) and implicit association tests (Bluemke \& Fiedler, 2009) decreased for frequent-frequent, as well as for infrequent-infrequent, combinations when the skew of stimulus base rates was manipulated.

However, despite this growing body of convergent evidence for bivariate PCs, there is no research so far on what may turn out to be the ultimate domain of PC inferences-namely, multicue tasks. Such inferences, which call for the parallel processing of several attributes and, therefore, must often proceed unconsciously (Shiffrin $\&$ Schneider, 1977), are crucial for adaptive learning in a complex probabilistic world (Chater \& Oaksford, 2008). Lie detection, for instance, is a biologically significant task that involves the simultaneous processing of such cues as gaze, posture, adapters, hesitations, and verbal indicators of truth (Vrij, 2008). Another basic multiple-cue module is reading, utilizing correlations among numerous graphical, orthographic, phonetic, and semantic cues. Whereas some paradigms merely call for passive assessment of cue intercorrelations, as in the present study, other paradigms include feedback for multicue inferences, such as diagnostic judgments (Hammond, 1955), risk assessment (Taylor, Hall, Cosier, \& Goodwin, 1996), and investment decisions (Slovic, 1969).

Organisms have an amazing ability to extract multiplecue correlations simultaneously. This assumption is crucial to Brunswik's (1943) probabilistic functionalism, which has inspired such important research programs as fast and frugal heuristics (Gigerenzer, Todd, \& the ABC Group, 1999), personality inferences from nonverbal cues (Gifford, 1994), and applied research on diagnostic and prognostic expert judgments. In all these paradigms, it has been taken for granted that organisms somehow manage to learn many criterion correlations and cue redundancies simultaneously. However, the theoretical question of what cognitive process can accomplish this monstrous task entails several unresolved puzzles. How can the complex data array needed to assess all $k \cdot(k-1) / 2$ contingencies between $k$ variables be administered in memory? How does the process handle the huge number of missing data that arise when, at every point in time, only a few of all $k$ attributes are available? When the $k$ attribute values belonging to the same individual $i$ are not observed jointly but are distributed over time and occasions, how can these wildly dispersed data be coordinated in memory?

PC inferences offer a simple solution of these unresolved puzzles, a viable alternative to the pragmatically impossible task of assessing a full correlation matrix. Rather than waiting for the completion of an ordered $n \times k$ matrix, the PC algorithm requires only cue base rates. When the distribution of a cue is skewed, showing a trend toward mostly high or mostly low values, detecting the modal value within several cue dimensions seems to be easy. Moreover, discerning cue base rates is possible regardless of missing data and regardless of whether the $k$ cues referring of the same individual $i$ appear jointly within the same block or are separated in a mixed sequence, even when the $k$ cues are presented on completely separate occasions.

Thus, studying PCs between multiple cues may provide a simple answer to an intriguing theoretical question. Given the constraints imposed on multiple-cue learning above, one might plausibly assume that PC inferences are more common in this paradigm than in the two-attribute paradigm in which they have been studied so far. However, what appears plausible still has to be verified empirically. To what extent the base rates of several cues can be assessed accurately and whether these base rates actually give rise to PC inferences that are strong enough to override actual correlations are open empirical questions. In the present study, we sought to demonstrate PCs under conditions that facilitated the assessment of genuine intercue correlations. On every observation trial, all $k$ cues of the same individual $i$ were presented simultaneously, thus providing wellcoordinated joint frequencies with which to compute correlations proper. Pairwise correlations were manipulated to either match or mismatch the PC imposed by the cue base rates. Our suspicion that it is not feasible to simultaneously assess $k \cdot(k-1) / 2$ correlations led us to expect only PC effects, unmoderated by the actual sign of correlations.

For a straightforward test of this working hypothesis, we employed a serial observation task without feedback. On every trial, a fictitious student was described by four attributes: gender (female vs. male), place (Mannheim vs. Heidelberg), subject matter (psychology vs. medicine), and hobby (sports vs. arts). The two levels within each dichotomous cue dimension always appeared at relative frequencies of $75 \%$ and $25 \%$, respectively, allowing for $4 \cdot 3 / 2=6$ PCs between pairs of cues. The actually presented cue correlations were manipulated to point in the same direction as the expected PCs or in the opposite direction, and some correlations were set to zero.

In this paradigm, the research strategy is to pit PCs against available correlation data, rather than providing no 
joint observations. Being exposed to all four attributes of each individual at the same time, participants have all the data necessary to assess the full $4 \times 4$ correlation matrix. However, even though all joint frequencies are available, the base rate or marginal frequencies are available too. Previous research has taken it for granted that correlation assessment uses joint frequencies (denoted $a, b, c, d$ for a standard $2 \times 2$ contingency matrix). However, the cognitive process may actually rely on the marginals, $(a+b)$, $(c+d),(a+c)$, and $(b+d)$, which inform PC inferences. If subsequent percentage judgments of each variable conditional on different levels of all other variables follow the $\mathrm{PC}$ rule, rather than the actual contingencies, and if this pattern comes along with support for the premise that the marginals are assessed more accurately than the joint frequencies, this would provide compelling evidence for PCs in a multiple-cue task setting.

\section{METHOD}

\section{Participants and Design}

Thirty-one male and female Heidelberg students participated for payment $(€ 3)$ or for course credit. They were randomly assigned to four different Latin square allocations of the four cue dimensions (gender, place, subject matter, and hobby, subsequently denoted G, P, $\mathrm{S}$, and $\mathrm{H}$, respectively) to the four rows and columns of the stimulus correlation matrix that defined the true intercue contingencies. Which level of each cue (e.g., the male or female level of G) appeared frequently ( $75 \%)$ and infrequently $(25 \%)$ was also varied. For convenience, let the sign of all contingencies be positive if it conforms to the PC prediction; all predicted PCs are thus, by definition, positive. The actually presented cue correlations were manipulated (Figure 1), such that four were approximately zero $(-.01<r<+.04)$, one correlation was positive or consistent with the PC $(r=+.31)$, and one was negative or inconsistent with the PC $(r=-.33)$.

\section{Materials and Procedure}

The entire experiment, including instructions, stimulus presentation, and dependent measures, was administered in a computer dialog. Instructions asked the participants to "discern regularities in an extended stimulus series." The participants were then presented with 100 stimulus displays, each providing four attributes of a target person in four differently colored rectangles arranged in a diamond, as in Figure 1. Although the colors of the rectangles in the four positions remained constant, the allocation of cues to colors/positions varied according to a Latin square. Displays were presented at a rate of $5 \mathrm{sec}$ per display, with a 2-sec interstimulus interval during which the screen remained light gray.

Immediately after the presentation stage, the participants were first asked to estimate the base rates of one focal value of each cue dimension. The judges typed in their percentage estimates for $\mathrm{G}$ (male), $\mathrm{H}$ (sports), $\mathrm{P}$ (Mannheim), and $\mathrm{S}$ (psychology) in a highlighted response window on the screen. To keep the number of judgments low, they were not asked to estimate the complementary (i.e., redundant) percentages for the nonfocal value (female, arts, Heidelberg, medicine). Afterward, contingency estimates were solicited in a similar format. For each pair of cues, the judges provided percentage estimates of the focal value of one cue, given both values of each other cue (e.g., "What \% of persons came from Mannheim given that they studied psychology?" and "What \% of people came from Mannheim given that they studied medicine?"). The order of judgments was constant across Latin square versions. At the end of the 15-min session, the participants were thanked and debriefed.

\section{RESULTS}

PC inferences rely on accurately assessed base rates. Confirming this premise, the objective base rate differences $\left(P_{\mathrm{o}}=75 \%\right.$ vs. $\left.25 \%\right)$ were readily extracted. Averaging across all cues, the mean subjective estimates $P_{\mathrm{s}}$ for the frequent and infrequent cue levels amounted to $69.9 \%$ and $30.1 \%$, respectively $[t(30)=9.40, p<.001]$. This base rate sensitivity held for all four cue dimensions [G (66.94\% vs. $30.33 \%), t=7.00 ; \mathrm{P}(72.38 \%$ vs. $34.87 \%)$, $t=8.63 ; \mathrm{S}(68.81 \%$ vs. $38.07 \%), t=5.92$; and $\mathrm{H}(71.38 \%$ vs. $31.13 \%), t=6.87$; all $p$ s $<.001]$.

To examine the premise that base rates are assessed more accurately than conditional rates, inaccuracy scores

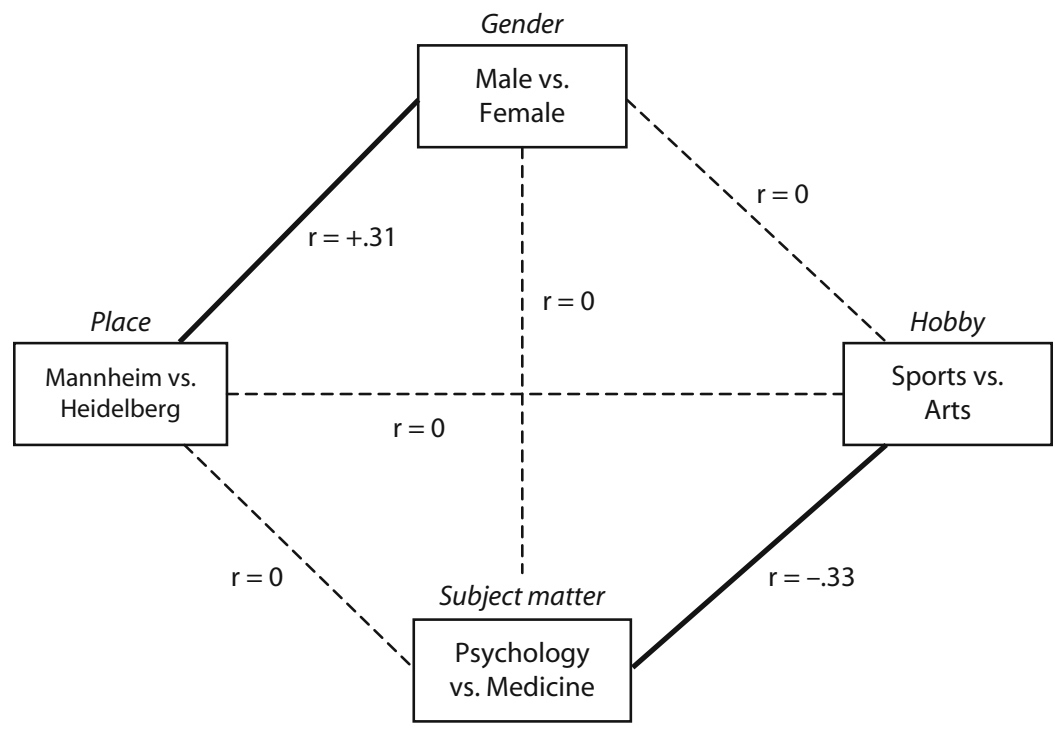

Figure 1. Pattern of negative, zero, and positive correlations between four dichotomous cues, used to demonstrate pseudocontingency inferences between multiple cues. (The figure represents one possible assignment of cue types to experimental roles.) 


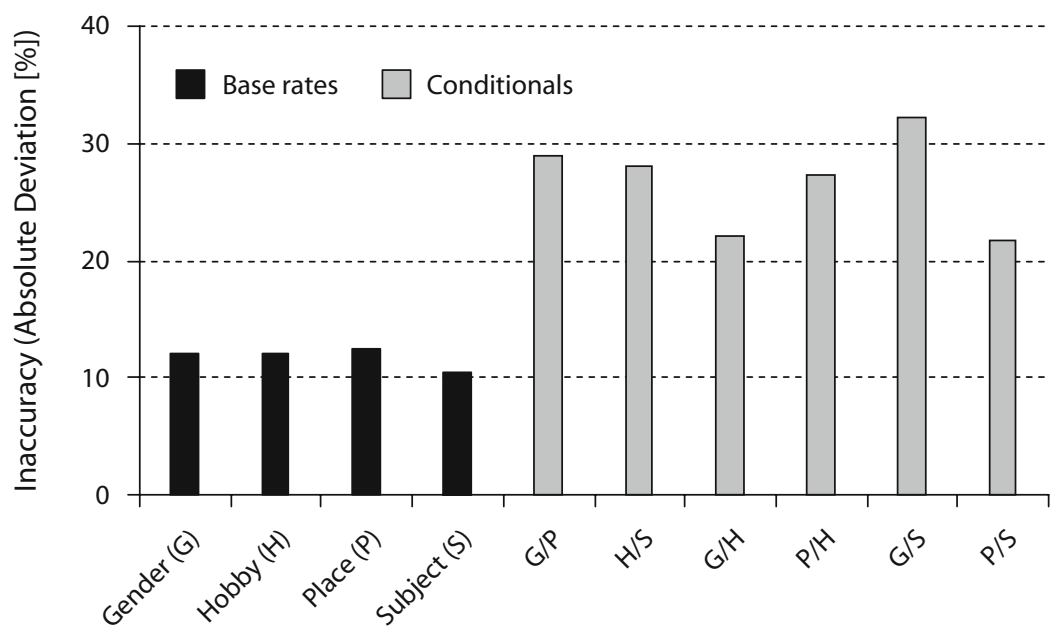

Figure 2. Mean inaccuracies of unconditional (base rate) and conditional percentage estimates.

(i.e., absolute differences $\left|P_{\mathrm{s}}-P_{\mathrm{o}}\right|$ between subjectively estimated and objectively presented percentages) were computed both for the base rate estimates and for the conditional estimates, averaging across the two conditional judgments required for each of the six pairwise contingencies [e.g., averaging the inaccuracy of the $P_{\mathrm{s}}$ (male $\mid$ psychology) and $P_{\mathrm{s}}$ (male $\mid$ medicine)]. Inaccuracy was systematically smaller for the base rates $(M=$ $11.82, S D=6.88)$ than for the conditionals defining the contingencies $(M=26.70, S D=9.94$; see Figure 2). A comparison of both sets of inaccuracies (averaging across the four base rate inaccuracies and the six pairs of conditional inaccuracies within each participant) yielded a significant $t(30)=6.85, p<.001$.

To examine the major hypothesis that PCs, rather than actually presented correlations, determine subjective contingency judgments, the two conditional estimates for each pair of cues were compared, as in a $\Delta_{p}$ measure. The expected PC effects would be supported if, across all attribute pairs $X, Y$, estimates of $P_{\mathrm{s}}$ (focal $Y$ value $\mid X$ value of matching frequency) were systematically higher than estimates of $P_{\mathrm{s}}$ (focal $Y$ value $\mid X$ value of mismatching frequency level). For example, if the focal gender "male" was presented frequently, and if the two places "Heidelberg" and "Mannheim" were presented frequently and infrequently, respectively, a PC would be reflected in a positive difference $P_{\mathrm{s}}$ (male $\mid$ Heidelberg $)-P_{\mathrm{s}}$ (male $\mid$ Mannheim). Whereas the PC hypothesis predicts a positive difference across all six pairs of cues, the zero contingency across the entire design (i.e., one positive, one negative, and four zero correlations) predicts no difference.

Across all 31 participants, the overall difference was positive for 21 , zero for 1 , and negative for 9 participants (binomial $p<.013$ ), suggesting a general PC trend across most participants. Testing the quantitative differences against zero yielded a significant $t(30)=2.77, p<.01$, when aggregating across all contingencies. When computed separately for the one negatively related pair $(t=$ $2.04, p=.05)$, the four zero correlations $(t=2.38, p<$
$.05)$, and the one positively related pair $(t=1.55$, n.s.), this PC effect hardly increased as a function of increasing actual correlations. The corresponding means are presented as black bars $\left(P_{\mathrm{s}}\right.$ for matching frequencies) and shaded bars ( $P_{\mathrm{s}}$ for mismatching frequencies) in Figure 3.

For a clear-cut demonstration that PCs override genuine contingencies, I finally included the component estimates, $P_{\mathrm{s}}$ (focal $Y$ value $\mid X$ value of matching frequency) and $P_{\mathrm{s}}$ (focal $Y$ value $\mid X$ value of mismatching frequency level), along with the true correlation factor $(r=-.33$ vs. 0 vs. +.31 ), in a $2 \times 3$ repeated measures ANOVA (averaging over conditional estimates of the four zerocorrelation conditions). The main effect of the first factor captures the PC. Any impact of the true correlation should be evident in an interaction.

The ANOVA results provided a clear-cut answer to the central research question. A significant PC main effect $\left[F(1,30)=7.60, p<.01, \eta^{2}=.253\right]$ reflected the fact that

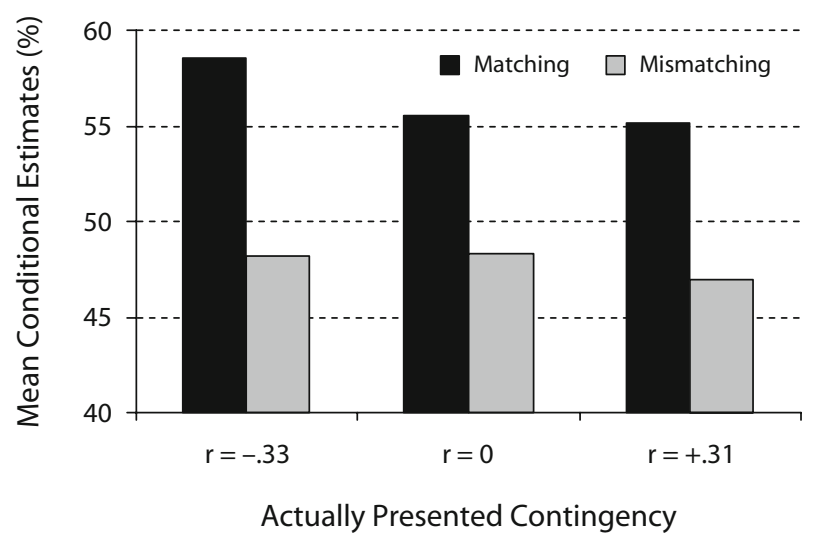

Figure 3. Mean percentage estimates of the focal value of one cue, given a matching frequency level (black bars) and given a mismatching frequency level (shaded bars) of other cues, averaged separately for cue pairs that were correlated negatively, at zero, or positively. 
the frequent level of one cue was associated more with the frequent than with the infrequent level of other cues. There was no sign of an interaction $\left[F(2,60)=0.15\right.$, n.s., $\eta^{2}=$ $.005]$, indicating that the size of the PC effect was unaffected by the true correlation (see Figure 3), although the statistical power was relatively high $[(1-\beta)=.725$; according to GPower; Faul, Erdfelder, Lang, \& Buchner, 2007]. Separate tests revealed that PC effects remained largely constant regardless of whether the actual correlation was PC inconsistent $[F(1,30)=4.16, p<.05]$, neutral $[F(1,30)=5.65, p<$ $.05]$, or PC consistent $[F(1,30)=2.39$, n.s.]. The latter condition actually produced a slightly weaker effect. Thus, subjective estimates followed the PCs derived from accurately assessed base rates, while being completely insensitive to actual correlations derived from the joint frequencies. (The lack of a main effect for true $r-F(2,60)=0.39$, n.s. -is theoretically meaningless.)

Consistent with this interpretation, those judges who most accurately extracted the four cue base rates fell prey to the strongest $\mathrm{PC}$ illusions (averaged across all six pairs; $r=+.43, p<.05)$. By complement, PC strength was negatively related to accurate assessment of conditional percentages $(r=-.34, p<.05)$.

\section{DISCUSSION}

As was predicted, PCs dominated genuine correlations in the present multiple-cue task. Although the participants were not deprived of genuine contingency input, since they received complete joint information about all four person attributes at the same time, their contingency estimates were exclusively driven by the cue base rates.

It is no surprise that as many as six contingencies cannot be assessed simultaneously from a single presentation of a multivariate series. Contingency assessment would amount to encoding and continuously updating six $2 \times 2$ schemes at the same time. To account for redundancies and partial correlations, it would even be necessary to keep separate records for any pair of variables at different levels of all the other variables. The present results highlight how unlikely it is that participants can keep in memory a blown-up contingency array or, alternatively, a high-fidelity exemplar-based record of all individual stimulus observations.

This unsurprising result was hardly considered in previous studies on multiple-cue learning (Lagnado, Newell, Kahan, \& Shanks, 2006; Shanks, 1990), inspired by the lens model (Brunswik, 1943), social judgment theory (Doherty \& Kurz, 1996), and cue redundancy effects (Garner, 1974). In virtually all of this prior work, it was presupposed that organisms extract correlations proper, on the basis of an exhaustive assessment of joint frequencies in a sample of stimulus observations. That there are alternative ways of inferring correlations or conditional probabilities has been discovered only very recently (Doherty, Anderson, Kelley, \& Albert, 2009; Fiedler et al., 2009; Zhao, Shah, \& Osherson, 2009).

We believe that PCs represent one such alternative inference route, which deserves to be further pursued in future research. When the assessment of individuating data is impossible, hard to encode, or unreliable, a viable strategy is to infer contingencies from ecological trends. If two high-frequency attributes tend to be jointly present in the same ecology, group, or category, or if two lowfrequency attributes tend to be jointly absent, the (Bayesian posterior) probability is high that this twofold trend reflects some genuine correlation.

Why do observers, or organisms more generally, rely on PCs, even in situations in which this inference is erroneous and illusory? A comprehensive answer to this crucial question is beyond the scope of this short research note (for a longer discussion, see Fiedler et al., 2009). Suffice it to point out that PCs are more than a worst-case remedy in the absence of more appropriate inferences. The wisdom of PC inferences is analogous to the lessons gained from research on probability matching and maximizing studies (Shanks, Tunney, \& McCarthy, 2002). In the probabilistic world, rather than trying to make a new prediction for each individual case, on the basis of whatever unreliable predictors happen to be available, a useful strategy is often to constantly predict the outcome that has the highest base rate in a given ecological context (Kareev, Fiedler, \& Avrahami, 2009). By extrapolation, such a strategy implies that, given multiple cues, organisms learn to expect jointly elevated (and jointly reduced) base rates in multicue environments. PCs arise as a natural by-product of this adaptive learning process. The PC illusion thus reflects the simultaneous utilization of several correlated base rate trends in particular ecologies, epochs, or stimulus contexts.

Where are the limits of PC inferences? To be sure, the most obvious precondition for inferring a PC between two attributes is that both base rate distributions must be sufficiently skewed. Only when there is a clear-cut trend toward mostly high or mostly low values for either attribute in an ecology can PC inferences link the frequent and the infrequent poles of both dimensions. An interesting implication of this restriction for the multicue domain is that the correlations for cues with skewed distributions should be easier to assess than correlations between equally distributed cue dimensions. Analogous to the way in which probability matching or maximizing strategies facilitate predictions for a single attribute whose outcome base rate is enhanced (Kareev et al., 2009), inferences about the relation between pairs of attributes are facilitated when one outcome is enhanced for either attribute.

Whether base rates are unequal or not, one should not overstate the case for PC dominance, expecting that proper contingency assessment is never possible. A century of research on conditioning affords ample evidence for humans' and animals' ability to learn from the joint occurrence of (present and absent) conditioned stimulus (CS) and unconditioned stimulus (US) events. The provision of a feedback or reinforcement that is contingent on a condition forces the organism to encode the pairing of CS and US, and such contingency learning should also be possible when CS and US base rates differ markedly from $50 \%$. Thus, an obvious implication that we are currently testing says that feedback-driven learning should render genuine contingency assessment more likely than passive observation. Nevertheless, even though actual contingen- 
cies may be easily extracted in a conditioning or feedback learning experiment, we have already obtained some evidence that PCs can intrude even into this home domain of contingency learning (Eder, Fiedler, \& Eder-Hamm, in press; Kutzner et al., 2008), moderating the success and speed of learning when one CS or predictor value and one US or criterion value is more frequent than the other.

Given that PC effects extend to simple feedback learning, in which the learner can focus on a single predictor, they can be expected to play an even stronger role in multiple-cue feedback learning. For example, when three cues or CSs compete for the prediction of a criterion or US, the learning of individual cue-criterion correlations may be blocked or overshadowed, but PC inferences may still be possible. In any case, theoretical predictions about multiple-cue feedback learning should be met with caution. Future research must figure out the relative contribution of PC inference and genuine contingency inference.

Finally, there is an intriguing rivalry at the theoretical level between PC inferences and exemplar-based inferences. The basic assumption of exemplar models of contingency learning (Juslin, Jones, Olsson, \& Winman, 2003) is that memory consists of separable entries for individual exemplars $i$ containing profiles of $k$ cues. Inferences about a new exemplar $j$ depend on the similarity, across subsets of the $k$ cues, between $j$ and all memorized exemplars (including $i$ ). The PC algorithm, in contrast, abstracts from individual exemplars, relying only on the cue base rates within a category or ecology. Unlike an exemplar-based memory code, the memory code used for PCs includes abstract cue base rates, which characterize the entire ecology (e.g., the stimulus list or the group from which individuals are drawn). Thus, exemplar-based memory strategies and category-based PC strategies delimit each other's domain. It can be suspected that exemplar-based inference strategies become more likely than category-based strategies to the extent that stimuli are wholistic and have a distinct identity and that the overall number of stimuli to be encoded is small or moderate. Specifying the precise boundaries between these two classes of models constitutes another important goal of future research.

\section{AUTHOR NOTE}

The research underlying the present article was supported by the Deutsche Forschungsgemeinschaft. Helpful comments on a draft of this article came from Thorsten Meiser, Tobias Vogel, and Peter Freytag. Correspondence concerning this article should be addressed to K. Fiedler, University of Heidelberg, Heidelberg, Germany (e-mail: kf@psychologie .uni-heidelberg.de).

\section{REFERENCES}

Bluemke, M., \& Fiedler, K. (2009). Base rate effects on the IAT. Consciousness \& Cognition, 18, 1029-1038.

BrunswiK, E. (1943). Organismic achievement and environmental probability. Psychological Review, 50, 255-272.

Chater, N., \& OAKsford, M. (Eds.) (2008). The probabilistic mind: Prospects for rational models of cognition. Oxford: Oxford University Press.

Doherty, M. E., Anderson, R. B., Kelley, A. M., \& Albert, J. H. (2009). Probabilistically valid inference of covariation from a single $x, y$ observation when univariate characteristics are known. Cognitive Science, 33, 183-205.
Doherty, M. E., \& Kurz, E. M. (1996). Social judgment theory. Thinking \& Reasoning, 2, 109-140.

Eder, A., Fiedler, K., \& Eder-Hamm, S. (in press). Illusory correlations revisited: The role of working memory capacity. Quarterly Journal of Experimental Psychology.

Faul, F., Erdfelder, E., Lang, A.-G., \& Buchner, A. (2007). G*Power 3: A flexible statistical power analysis program for the social, behavioral, and biomedical sciences. Behavior Research Methods, 39, 175-191.

Fiedler, K., \& Freytag, P. (2004). Pseudocontingencies. Journal of Personality \& Social Psychology, 87, 453-467.

Fiedler, K., Freytag, P., \& Bluemke, M. (2010). Pseudocontingencies in evaluative priming. Unpublished research, University of Heidelberg.

Fiedler, K., Freytag, P., \& Meiser, T. (2009). Pseudocontingencies: An integrative account of an intriguing cognitive illusion. Psychological Review, 116, 187-206.

Fiedler, K., Freytag, P., \& Unkelbach, C. (2007). Pseudocontingencies in a simulated classroom. Journal of Personality \& Social Psychology, 92, 665-677.

Freytag, P. (2003). Contextually determined typicality. Berlin: Logos.

Freytag, P., Kutzner, F., Vogel, T., \& Fiedler, K. (2010). On the adaptive value of pseudocontingencies. Unpublished research, University of Heidelberg.

GARnER, W. R. (1974). The processing of information and structure. Potomac, MD: Erlbaum.

GIFFORD, R. (1994). A lens-mapping framework for understanding the encoding and decoding of interpersonal dispositions in nonverbal behavior. Journal of Personality \& Social Psychology, 66, 398-412.

Gigerenzer, G., TodD, P. M., \& The ABC Group (1999). Simple heuristics that make us smart: Evolution and cognition. New York: Oxford University Press.

Hammond, K. R. (1955). Probabilistic functioning and the clinical method. Psychological Review, 62, 255-262.

Juslin, P., Jones, S., Olsson, H., \& Winman, A. (2003). Cue abstraction and exemplar memory in categorization. Journal of Experimental Psychology: Learning, Memory, \& Cognition, 29, 924-941.

Kareev, Y., Fiedler, K., \& Avrahami, J. (2009). Base rates, contingencies, and prediction behavior. Journal of Experimental Psychology: Learning, Memory, \& Cognition, 35, 371-380.

Kutzner, F., Freytag, P., Vogel, T., \& Fiedler, K. (2008). Base-rate neglect as a function of baserates in probabilistic contingency learning. Journal of the Experimental Analysis of Behavior, 90, 23-32.

Lagnado, D. A., Newell, B. R., Kahan, S., \& Shanks, D. R. (2006). Insight and strategy in multiple-cue learning. Journal of Experimental Psychology: General, 135, 162-183.

Meiser, T., \& Hewstone, M. (2004). Cognitive processes in stereotype formation: The role of correct contingency learning for biased group judgments. Journal of Personality \& Social Psychology, 87, 599-614.

Shanks, D. R. (1990). Connectionism and the learning of probabilistic concepts. Quarterly Journal of Experimental Psychology, 42A, 209237.

Shanks, D. R., Tunney, R. J., \& McCarthy, J. D. (2002). A reexamination of probability matching and rational choice. Journal of Behavioral Decision Making, 15, 233-250.

ShIFFrIN, R. M., \& SchneIDER, W. (1977). Controlled and automatic human information processing: II. Perceptual learning, automatic attending, and a general theory. Psychological Review, 84, 127-190.

SLovic, P. (1969). Analyzing the expert judge: A descriptive study of a stockbroker's decision process. Journal of Applied Psychology, 53, 255-263.

Taylor, L., Hall, P., Cosier, R., \& Goodwin, V. (1996). Outcome feedback effects on risk propensity in an MCPLP task. Journal of Management, 22, 299-311.

VRIJ, A. (2008). Detecting lies and deceit: Pitfalls and opportunities (2nd ed.). New York: Wiley.

Zhao, J., Shah, A., \& Osherson, D. (2009). On the provenance of judgments of conditional probability. Cognition, 113, 26-36.

(Manuscript received July 7, 2009;

revision accepted for publication January 26, 2010.) 